\title{
Langzeit-Compliance der CPAP-Therapie - aktueller Stand, Prädiktoren und Interventionsmöglichkeiten
} Long-term Compliance of CPAP-Therapy - Update, Predictors and Interventions

Autoren

Institut
M. Orth ${ }^{1}$, S. Kotterba ${ }^{2}$, J. W. Walther ${ }^{1}$, K. Rasche ${ }^{3}$, G. Schultze-Werninghaus ${ }^{1}$, H.-W. Duchna ${ }^{1}$

Berufsgenossenschaftliche Kliniken Bergmannsheil, Universitätsklinik, Klinikum der Ruhr-Universität Bochum, Medizinische Klinik III, Pneumologie, Allergologie, Schlaf- und Beatmungsmedizin

Neurologische Klinik und Poliklinik

Kliniken St. Antonius, Wuppertal eingereicht 3.42006

akzeptiert 9.52006

\section{Bibliografie}

DOI $10.1055 / \mathrm{s}-2006-944234$

Pneumologie 2006; 60;

480-484

(c) Georg Thieme Verlag KG Stuttgart · New York .

ISSN 0934-8387

Korrespondenzadresse Priv.-Doz. Dr. Maritta Orth BG-Kliniken Bergmannsheil, Universitätsklinik · Klinikum der Ruhr-Universität Bochum, Medizinische Klinik III, Pneumologie, Allergologie, Schlaf- und Beatmungsmedizin Bürkle-de-la-Camp-Platz 1 44789 Bochum maritta.orth@rub.de

\section{Zusammenfassung}

CPAP (continuous positive airway pressure) ist die Therapie der Wahl des obstruktiven Schlafapnoe-Syndroms (OSAS). Ca. 70\% der Patienten mit eingeleiteter CPAP-Therapie nutzen das Gerät dauerhaft während 70\% der Nächte für mindestens 4 Stunden pro Nacht. Prädiktoren für eine gute CPAP-Langzeitcompliance sind der Schweregrad der Atmungsstörung bzw. der Störung der Schlafarchitektur, der Ausprägungsgrad der Tagesmüdigkeit sowie die Patientenzufriedenheit mit dem diagnostischen und therapeutischen Procedere. Eine Steigerung der CPAP-Compliance ist durch intensive Patientenschulung zu erzielen. Apparative Alternativen zu CPAP wie z. B. Bilevel oder auto-CPAP-Systeme können die Langzeitcompliance nicht steigern.

\section{Einleitung}

Die continuous positive airway pressure (CPAP)Therapie, die 1981 von Collin Sullivan inauguriert wurde, ist nach wie vor der Goldstandard in der Behandlung des mittel- bis schwergradigen obstruktiven Schlafapnoe-Syndroms (OSAS). Hinsichtlich der Nutzung der Geräte müssen grundsätzlich zwei Faktoren unterschieden werden: die Akzeptanz, d.h. die primäre Annahme des Gerätes in der unmittelbaren Einstellungssituation im Schlaflabor und den Willen, das Gerät in der häuslichen Umgebung anzuwenden und die im Hinblick auf die Beurteilung des Therapieerfolges entscheidendere Langzeit-Compliance, d.h. die dauerhafte Nutzung des Gerätes in der häuslichen Umgebung [1]. Hierbei stellt sich grundsätzlich die Frage, wie sich die Compliance der CPAP-Therapie definiert. Unklar ist nach wie vor,

\section{Abstract}

CPAP (continuous positive airway pressure) is the therapy of choice for obstructive sleep apnea syndrome (OSAS). About $70 \%$ of patients on CPAP use their device for at least $70 \%$ of nights with an application-time of at least 4 hours per night. Severity of breathing disorder respectively disturbance of sleep architecture, degree of daytime sleepiness and patients' satisfaction with diagnostic and therapeutic procedures are good predictors of long-term CPAP-compliance. CPAPcompliance can be increased by intensive patient education. Alternative devices like for example Bilevel or auto-CPAP respectively cannot augment CPAP compliance. wie viel CPAP-Therapie der jeweilige Patient benötigt. Entscheidend für die Langzeit-Compliance scheint die suffiziente Besserung der Folgeerscheinungen des OSAS und der Tagessymptomatik in Form der ausgeprägten Tagesmüdigkeit zu sein.

Die CPAP-Therapie stellt keine für den Patienten nebenwirkungsfreie Therapie dar. Tab. 1 zeigt eine Aufstellung der in der Literatur beschriebenen Nebenwirkungen der CPAP-Therapie. Beklagt werden vor allem Probleme, die im Zusammenhang mit der Anwendung der CPAP-Maske stehen. Hierzu gehören das allgemeine Missempfinden beim Tragen der Maske (50\% der Patienten), trockene Nasenschleimhäute $(65 \%$ der Patienten), Nasenlaufen sowie Stockschnupfen (40\% der Patienten). Zum Teil können diese Probleme durch Applikation von Warmluftbefeuchtern behoben werden. 
Tab. 1 Art und Häufigkeit von Nebenwirkungen der CPAP-Therapie im Langzeitverlauf (modifiziert nach [40])

\begin{tabular}{|c|c|c|}
\hline Beschwerden & $\begin{array}{l}\text { Häufigkeit } \\
\text { (\%) }\end{array}$ & Autoren \\
\hline $\begin{array}{l}\text { Nebenwirkungen } \\
\text { allgemein: }\end{array}$ & $43-88 \%$ & $\begin{array}{l}\text { Waldhorn u. Mitarb. } 1990 \text { [41] } \\
\text { Akashida u. Mitarb. } 1992 \text { [42] } \\
\text { Pépin u. Mitarb. } 1995 \text { [43] }\end{array}$ \\
\hline $\begin{array}{l}\text { Beeinträchtigung } \\
\text { durch Geräusch- } \\
\text { entwicklung }\end{array}$ & $15-60 \%$ & $\begin{array}{l}\text { Waldhorn u. Mitarb. } 1990 \text { [41] } \\
\text { Hoffstein u. Mitarb. } 1992 \text { [44] } \\
\text { Orth u. Mitarb. } 1993 \text { [2] } \\
\text { Engleman u. Mitarb. } 1996 \text { [45 }\end{array}$ \\
\hline Maskenprobleme & $24-66 \%$ & $\begin{array}{l}\text { Waldhorn u. Mitarb. } 1990 \text { [41] } \\
\text { Akashida u. Mitarb. } 1992 \text { [42] } \\
\text { Hoffstein u. Mitarb. } 1992 \text { [44] } \\
\text { Orth u. Mitarb. } 1993 \text { [2] } \\
\text { Pépin u. Mitarb. } 1995 \text { [43] } \\
\text { Engleman u. Mitarb. } 1996 \text { [45 }\end{array}$ \\
\hline $\begin{array}{l}\text { Eintrocknung der } \\
\text { Nasenschleim- } \\
\text { häute, Rhinitis }\end{array}$ & $30-70 \%$ & $\begin{array}{l}\text { Waldhorn u. Mitarb. } 1990 \text { [41] } \\
\text { Akashida u. Mitarb. } 1992 \text { [42] } \\
\text { Hoffstein u. Mitarb. } 1992 \text { [44] } \\
\text { Orth u. Mitarb. } 1993 \text { [2] } \\
\text { Engleman u. Mitarb. } 1996 \text { [45 }\end{array}$ \\
\hline $\begin{array}{l}\text { Reizungen der } \\
\text { Augen }\end{array}$ & $1-24 \%$ & $\begin{array}{l}\text { Waldhorn u. Mitarb. } 1990 \text { [41] } \\
\text { Hoffstein u. Mitarb. } 1992 \text { [44] } \\
\text { Orth u. Mitarb. } 1993 \text { [2] } \\
\text { Pépin u. Mitarb. } 1995 \text { [43] } \\
\text { Engleman u. Mitarb. } 1996 \text { [45 }\end{array}$ \\
\hline
\end{tabular}

\section{Complianceraten}

Erste Studien zum Thema Langzeit-Compliance der CPAP-Therapie datieren aus den frühen 80er-Jahren und basieren überwiegend auf Patientenangaben. Tab. 2 zeigt eine Zusammenstellung der Complianceraten anhand der Literaturangaben. Fasst man die Studienlage zusammen, so ist davon auszugehen, dass ca. $70 \%$ der Patienten das Gerät während mindestens 70\% der Nächte für mindestens 4 Stunden/Nacht benutzen. Im Rahmen einer eigenen Untersuchung konnte anhand der anamnestischen Angaben der Patienten eine Langzeitcompliance von 78\% Nutzung des Gerätes während mindestens 5 Stunden pro Nacht ermittelt werden [2]. Schon früh wurde allerdings nachgewiesen, dass die subjektiven Patientenangaben die tatsächliche Nutzung um mindestens eine Stunde überschätzen. So konnten Rauscher u. Mitarb. zeigen, dass die tatsächliche Nutzung, ermittelt anhand von Betriebsstundenzählern, 4,9 $\pm 0,3$ Stunden betrug und somit die im gleichen Kollektiv von Patienten mit schwergradigem OSAS (AHI 50,8 $\pm 2,9 / \mathrm{h}$ ) subjektive Einschätzung der Nutzung mit 6,1 $\pm 0,3$ Stunden pro Nacht um mehr als eine Stunde unterschritt [3]. Diese Ergebnisse wurden bestätigt von Reeves-Hoché u. Mitarb., die ebenfalls in einem Nachbeobachtungszeitraum von 6 Monaten nach eingeleiteter CPAP-Therapie anhand der Stundenzähler eine effektive Nutzung von 4,3 Stunden pro Nacht nachweisen konnten [4].

\section{Gibt es Prädiktoren für die Langzeit-Compliance?}

Die CPAP-Therapie führt insbesondere bei Patienten mit schwergradigem Apnoebefund und ausgeprägter Tagessymptomatik zu einer raschen Besserung der geklagten Beschwerden. Insofern stellt die Kombination aus ausgeprägter Tagessymptomatik, gemessen z.B. am Epworth Sleepiness Score (ESS) und mittel- bis schwergradig ausgeprägtem Apnoe-Hypopnoe-Index (AHI) einen wesentlichen Prädiktor für eine dauerhafte CPAP-Nutzung dar. Tab. 3 zeigt eine Zusammenstellung von Studien, in denen Prädiktoren für die dauerhafte Anwendung des CPAP-Gerätes analysiert werden. Krieger u. Mitarb. konnten nachweisen, dass der AHI entscheidend ist für die dauerhafte Nutzung des Gerätes [5]. Patienten mit einem AHI > 15/h zeigten über einen follow-up von über 3 Jahren eine signifikant bessere Langzeitcompliance als solche mit einem $\mathrm{AHI}<15 / \mathrm{h}$. Auch lag die Dauer der Nutzung mit 5,7 $\pm 1,8$ Stunden pro Nacht (Stundenzähler) deutlich über den von Reeves-Hoché und Rauscher ermittelten Angaben [3,4]. McArdle u. Mitarb. zeigten, dass Patienten mit einer Kombination aus ESS-Score $>10$ und AHI $>30 /$ h eine signifikant bessere Langzeit-Compliance aufweisen als solche, die diese Voraussetzung nicht erfüllen [6]. Entscheidend für die dauerhafte Nutzung ist hierbei nicht der Schwergrad der Atmungsstörung, sondern vielmehr der Ausprägungsgrad der Einschränkungen der Tagesbefindlichkeit: Patienten mit einem höhergradigen AHI nutzten die Therapie wesentlich schlechter bei Vorliegen eines ESS-Scores $<10$. Die Bedeutung der Tagesmüdigkeit für die regelmäßige und dauerhafte Gerätenutzung belegt ebenso die Untersuchung von Sin u. Mitarb. Weiterhin scheint das Patientenalter einen Einfluss auf die Langzeit-Compliance auszuüben: im höheren Lebensalter steigt die Compliance $[7,8]$.

Daneben stellt die Störung des Schlafprofils bzw. dessen Besserung unter CPAP ebenfalls einen entscheidenden Faktor für die Langzeit-Compliance dar. In der Untersuchung von Drake u. Mit-

\begin{tabular}{|c|c|c|c|c|}
\hline Autor & Art d. Registrierung & Nachbeobachtung & Nutzungsdauer & \multirow{9}{*}{$\begin{array}{l}\text { Tab. } 2 \text { Langzeit-Compliance- } \\
\text { raten in der Literatur }\end{array}$} \\
\hline Barnes u. Mitarb. 2002 [46] & Stundenzähler & 8 Wochen & $3,53 \pm 2,1 \mathrm{~h} / \mathrm{Tag}$ & \\
\hline Krieger u. Mitarb. 1996 [7] & Stundenzähler & $1176 \pm 38$ Tage & $\begin{array}{l}90 \% \text { d. Pat. } \\
5,7 \pm 2,8 \mathrm{~h} / \mathrm{Tag}\end{array}$ & \\
\hline Sin u. Mitarb. 2002 [8] & monitoring chip & 6 Monate & $\begin{array}{l}88,5 \% \text { d. Pat. } \\
5,8 \text { h/Tag }\end{array}$ & \\
\hline Rauscher u. Mitarb. 1993 [3] & Stundenzähler & $539 \pm 44$ Tage & $\begin{array}{l}71 \% \text { d. Pat. } \\
4,9 \pm 0,3 \mathrm{~h} / \text { Tag }\end{array}$ & \\
\hline Engleman u. Mitarb. 1994 [47] & Stundenzähler & 3 Monate & $\begin{array}{l}60 \% \text { d. Pat. } \\
4,7 \pm 0,4 \mathrm{~h} / \mathrm{Nacht}\end{array}$ & \\
\hline Reeves-Hoche u. Mitarb. 1994 [4] & Stundenzähler & 3 Monate & $\begin{array}{l}80 \% \text { d. Pat. } \\
4.3 \mathrm{~h} / \text { Nacht }\end{array}$ & \\
\hline Pépin u. Mitarb. 1999 [48] & Stundenzähler & 3 Monate & $\begin{array}{l}79 \% \text { d. Pat. } \\
\geq 4 \mathrm{~h} / \text { Nacht }\end{array}$ & \\
\hline Pépin u. Mitarb. 1995 [42] & Stundenzähler & $19 \pm 17$ Monate & $\begin{array}{l}88 \% \text { d. Pat. } \\
6,5 \pm 3 \mathrm{~h} / \mathrm{Nacht}\end{array}$ & \\
\hline
\end{tabular}




\begin{tabular}{|c|c|c|}
\hline & Autor & Compliance-Prädiktor \\
\hline Atmungsstörung & $\begin{array}{l}\text { Hui u. Mitarb. } 2001 \text { [49] } \\
\text { Krieger u. Mitarb. } 1996 \text { [7] } \\
\text { Mc Ardle u. Mitarb. } 1999 \text { [6] } \\
\text { Meurice u. Mitarb. } 1994 \text { [50] }\end{array}$ & $\begin{array}{l}\mathrm{AHI} \\
\mathrm{AHI}>15 / \mathrm{h} \\
\mathrm{AHI}>30 / \mathrm{h} \\
\mathrm{AHI}\end{array}$ \\
\hline Schlafstörung & $\begin{array}{l}\text { Meurice u. Mitarb. } 1994 \text { [50] } \\
\text { Drake u. Mitarb. } 2003 \text { [9] }\end{array}$ & $\begin{array}{l}\text { Leicht- und Tiefschlafanteil } \\
\text { Besserung d. Schlafeffizienz }\end{array}$ \\
\hline Tagesmüdigkeit & $\begin{array}{l}\text { Mc Ardle u. Mitarb. } 1999 \text { [6] } \\
\text { Sin u. Mitarb. } 2002[8]\end{array}$ & ESS $>10$ \\
\hline Diagnostik/Therapie & $\begin{array}{l}\text { Krieger u. Mitarb. } 1998 \text { [10] } \\
\text { Means u. Mitarb. } 2004 \text { [11] }\end{array}$ & $\begin{array}{l}\text { polysomnographische Diagnostik } \\
\text { polysomnographische CPAP-Titration }\end{array}$ \\
\hline andere & $\begin{array}{l}\text { Krieger u. Mitarb. } 1996 \text { [7] } \\
\text { Sin u. Mitarb. } 2002 \text { [8] } \\
\text { Popescu u. Mitarb. } 2001 \text { [13] } \\
\text { Ball u. Mitarb. } 2001 \text { [14] }\end{array}$ & $\begin{array}{l}\text { hohes Alter, hoher BMI } \\
\text { höheres Alter, weibl. Geschlecht } \\
\text { Patientenzufriedenheit } \\
\text { Patientenzufriedenheit }\end{array}$ \\
\hline
\end{tabular}

Tab. 3 Prädiktoren der CPAPCompliance arb. war die Besserung der Schlafeffizienz in der Einstellungsphase auf die CPAP-Therapie der wesentliche Prädiktor für eine dauerhafte Therapienutzung und führte bei den Patienten mit akut gebesserter Schlafeffizienz zu einer 2 Stunden längeren CPAP-Applikation pro Nacht als bei den Patienten, bei denen die Schlafeffizienz akut nicht gebessert werden konnte [9].

In zunehmendem Maße wird versucht, die Schlafmedizin in den ambulanten Bereich zu überführen. Neben der Kostenersparnis für die jeweiligen Leistungsträger wird als Argument für das ambulante Vorgehen häufig auch die Steigerung der Akzeptanz durch den Patienten angeführt, dem mittels der ambulanten Diagnostik und ggf. auch Therapie ein Krankenhausaufenthalt erspart bleibt. Dass das ambulante Vorgehen bereits im Zuge der Vordiagnostik sich entscheidend auf die Langzeit-Compliance auswirken kann, zeigt die Untersuchung von Krieger u. Mitarb. [10]. Hier wurde bei jeweils 60 Patienten die Diagnose des OSAS entweder polygraphisch (MESAM IV) oder polysomnographisch gestellt. Die CPAP-Anpassung erfolgte unter Laborbedingungen polysomnographisch. Es konnte über einen Beobachtungszeitraum von 2 Jahren nachgewiesen werden, dass die Ausfallquote bei den per MESAM diagnostizierten Patienten signifikant höher (21,7\% MESAM vs. 10,0\% PSG, p < 0,05) und die CPAP-Nutzungsdauer deutlich kürzer war als bei den Patienten, bei denen bereits die Diagnosestellung polysomnographisch erfolgte $(4,3 \pm$ 0,3 h/Nacht MESAM vs.5,3 $\pm 0,2 \mathrm{~h} /$ Nacht PSG, $\mathrm{p}<0,05$ ). Auch in neueren Untersuchungen konnte die Überlegenheit der LaborCPAP-Titration gegenüber der Einstellung im häuslichen Bereich im Hinblick auf die Langzeitcompliance belegt werden [11]. Ebenso steigern die Patientenzufriedenheit mit der Diagnostik und der Therapie die Langzeitanwendung von CPAP [12,13].

Die umgekehrte Frage nach negativen Prädiktoren für die Langzeit-CPAP-Compliance bestätigt die vorgenannten Fakten: So wirken sich ein Body-Mass-Index (BMI) $\leq 30 \mathrm{~kg} / \mathrm{m}^{2}$, ein ESS-Score $\leq 15$, ein $A H I \leq 30 / h$ und ein CPAP-Druck $\geq 12$ mbar negativ auf die dauerhafte CPAP-Nutzung aus [37]. Kontrovers in der Literatur wird der Einfluss des Geschlechts auf die CPAP-Compliance diskutiert $[8,14]$, nach eigenen Erfahrungen ist die primäre Akzeptanz der CPAP-Therapie bei Frauen geringer als bei Männern, bezüglich der Compliance erweisen sich Frauen jedoch als mindestens so therapietreu wie Männer. Weiterhin beeinflussen negative Erfahrungen mit dem CPAP-Gerät in der Initialphase der Anwendung, einschneidende Lebensereignisse wie z.B. Partnerverlust sowie das Alleinleben die Compliance negativ [15]. Nahezu unmöglich ist die Einleitung einer CPAP-Therapie bei Vorliegen einer Klaustrophobie [16].

\section{Wie können wir die CPAP-Compliance steigern?}

Die Frage, wie die CPAP-Compliance bzw. die Patientenmotivation zu steigern sei, ist Gegenstand einer 2005 erschienenen Cochrane-Analyse [17]. In dieser Untersuchung wurden 24 randomisierte kontrollierte Studien mit insgesamt 1007 Patienten mit der Frage analysiert, ob die Patientencompliance durch mechanische Interventionen (z.B. Bilevel-Therapie, auto-CPAP) bzw. durch Schulungsmaßnahmen zu steigern sei.

\section{Patientenschulung}

Eine frühe Untersuchung zum Thema Steigerung der Langzeitcompliance durch intensive Patientenschulung in Form von Unterricht, Videodemonstration und zusätzlichen regelmäßigen Telefonkontakten verglichen mit fehlender Patientenschulung in einem cross-over Design erbrachte wenig ermutigende Ergebnisse: die Compliance der CPAP-Therapie unterschied sich nicht zwischen beiden Kollektiven [18]. Demgegenüber liegen heute neuere Studien vor, die belegen, dass die intensive Schulung der Patienten z. B. durch Videomaterial [19] oder Verhaltenstherapie [20] die Langzeitcompliance signifikant zu steigern vermag. Ebenso kann die Compliance durch zusätzliche Schulung in der häuslichen Umgebung unter Miteinbeziehung des jeweiligen Partners die Langzeitakzeptanz signifikant steigern [21]. Auch scheint die Überlassung von schriftlichem Informationsmaterial effektiver zu sein als regelmäßige Telefonkontakte, denn sie steht sowohl dem Patienten als auch seinen Angehörigen zur Information jederzeit zur Verfügung [22].

\section{Apparative Maßnahmen}

\section{Masken und Befeuchter}

Die mit der CPAP-Therapie einhergehenden Nebenwirkungen betreffen vor allem den Tragekomfort der Maske sowie die lokalen Auswirkungen des Luftstromes auf die Schleimhäute der oberen Atemwege. Alternativ zu den üblicherweise primär eingesetzten konfektionierten Nasenmasken stehen Mundmasken, Gesichtsmasken bzw. sog. „nasal pillows“ (Nasenstöpsel) zur Verfügung. Weder durch Applikation von Mundmasken bzw. Nasenstöpseln kann anhand der Studienlage die Langzeitnutzung eindeutig gesteigert werden, allenfalls sind lokale Nebenwirkungen (z. B. Undichtigkeiten) im Einzelfall besser handhab- 
bar [23,24]. Die möglichst optimale Anpassung der Maske an die individuellen Bedürfnisse und mit dem Ziel, einen optimalen Tragekomfort zu erreichen, stellt dennoch eine der wichtigsten Herausforderungen an den behandelnden Schlafmediziner dar. Im Einzelfall ist hierbei auch die Anfertigung einer individuellen Maske zu erwägen.

Die Austrocknung der Nasenschleimhäute ist mithilfe von Luftbefeuchtern gut behandelbar, wobei sich Kaltluftbefeuchter als ineffektiv erwiesen haben [25]. Hingegen kann mithilfe einer Warmluftbefeuchtung sowohl eine Reduktion der lokalen Nebenwirkungen $[25,26]$ als auch in eine Steigerung der Langzeitcompliance erreicht werden [26].

\section{Geräte}

Die initialen Probleme der CPAP-Therapie, die mit der bedingt durch die Größe schweren Handhabbarkeit bzw. der Lautstärke assoziiert waren, sind inzwischen weitgehend gelöst. Mittlerweile stehen kleine, selbst auf Reisen anwendbare und in ihrer Lautstärke sowohl den Patienten als auch den Schlafpartner kaum noch belästigende Systeme zur Verfügung.

Schon früh wurde vor dem Hintergrund, dass der positive inspiratorische Druck nur in der Einatmungsphase relevant ist und bei manchen Patienten nur in bestimmten Körperlagen Apnoen bzw. Hypopnoen auftreten, so genannte auto-CPAP-Geräte entwickelt, die bedarfsweise, anhand unterschiedlicher Algorithmen den CPAP-Druck steigern und, falls nicht mehr erforderlich, absenken können. Die Erwartung war, auf der Basis einer lediglich „bedarfsorientierten“ Druckapplikation eine höhere Therapieakzeptanz und eine Steigerung der Langzeitcompliance zu erreichen.

Die Studienlage zum Thema „Langzeitakzeptanz CPAP vs. autoCPAP“ muss grundsätzlich in zwei verschiedene Studientypen eingeteilt werden:

- Studien mit randomisiertem, parallelem Design

- Studien mit randomisiertem, doppelblindem cross-over-Design.

Die Ergebnisse der Untersuchungen, die im randomisierten parallelen Design durchgeführt wurden, zeigen kontroverse Ergebnisse bezüglich der Langzeitcompliance. Ficker u. Mitarb. sahen bei jeweils 50 Patienten keinen Unterschied in der Anwendungsdauer [27]. Hingegen wiesen Meurice u. Mitarb. bei identischer Besserung der klinischen Symptome eine längere Nutzungsdauer unter auto-CPAP $(6,5 \pm 1,0 \mathrm{~h} / \mathrm{Nacht}$ auto-CPAP vs. $5,1 \pm$ 1,1 h/Nacht CPAP, $\mathrm{p}=0,02$ ) nach, allerdings beschränkte sich der Beobachtungszeitraum auf drei Wochen [28]. Konermann u. Mitarb. fanden bei 50 Patienten mit OSAS in einem follow-up von 6 Monaten zwar keine Unterschiede der Nutzungsdauer pro Nacht, jedoch nutzten Patienten mit auto-CPAP häufiger das Gerät bezogen auf die Anzahl der Nutzungsnächte pro Woche $(6,5 \pm 0,4$ Nächte/Woche auto-CPAP vs. 5,7 $\pm 0,7$ Nächte/Woche CPAP, $\mathrm{p}<0,01$ ) [29].

Die Studien zum Thema auto-CPAP vs. CPAP, die im randomisierten cross-over- Design durchgeführt wurden, zeigen überwiegend keinen Vorteil im Hinblick auf die Langzeit-Compliance $[30,31,32]$. Lediglich in zwei Untersuchungen war die Nutzungsdauer pro Nacht um 30 Minuten länger als unter CPAP $[33,34]$. Auch der Vergleich von konventionellem CPAP gegenüber der Anwendung einer Bilevel-Therapie zeigt keine Unterschiede im Hinblick auf die Langzeitcompliance [35,36]. Vielversprechend, wenngleich auch nur in einem Beobachtungszeitraum von drei Monaten, scheint die Anwendung von C-Flex. In einer Untersuchung konnten Aloia u. Mitarb. bei Patienten, die mit C-Flex be- handelt wurden, gegenüber konventionellem CPAP eine signifikant längere Nutzungsdauer nachweisen $(4,8 \pm 2,4 \mathrm{~h} / \mathrm{Nacht}$ C-Flex vs. 3,1 $\pm 2,8 \mathrm{~h} /$ Nacht CPAP) [20].

\section{Ausblick}

Die Ergebnisse der Cochrane-Analyse sind für den Schlafmediziner ermutigend. Sie zeigen, dass nach wie vor der Mensch, in diesem Fall das Personal der schlafmedizinischen Abteilung, die wichtigste Rolle hat, wenn es um die Motivation zur dauerhaften Nutzung des CPAP-Gerätes geht. Hierzu sind ärztliches Einfühlungsvermögen, Geduld und ein enger Kontakt zwischen schlafmedizinischer Abteilung und Patient notwendig. Neben der erforderlichen schlafmedizinischen Kompetenz unterstreicht die Analyse aber auch den positiven Einfluss der stationären schlafmedizinischen Diagnostik und Therapie im Vergleich zum ambulanten Vorgehen im Hinblick auf die CPAP-Langzeitcompliance: der im stationären Setting diagnostizierte und behandelte Patient nimmt seine Erkrankung und die erforderliche Therapie ernster. Der hierfür erforderliche Zeitaufwand muss sich zukünftig in den Leistungskatalogen widerspiegeln. Denkbare weitere, wenngleich nicht an größeren Patientenkollektiven evaluierte Motivationsfaktoren, sind die intensive Aufklärung über das erhöhte Unfallrisiko sowie die drohenden kardiovaskulären Ereignisse $[37,38,39]$ bei unbehandeltem schwerem OSAS sowie der Einfluss von Intelligenz bzw. psychologischen Faktoren wie Angst und Depression auf die dauerhafte CPAP-Nutzung.

\section{Literatur}

1 Anstead M, Phillips B, Buch K: Tolerance and intolerance to continuous positive airway pressure. Curr Opin Pulm Med 1998; 4: 351 - 354

2 Orth $M$, Rasche $K$, Ullrich HUet al: Long-term compliance of n-CPAP therapy by patients with sleep related respiratory disorders. Pneumologie 1995; 49 (Suppl.1): 121 - 125

3 Rauscher H, Formanek D, Popp Wet al: Self-reported vs measured compliance with nasal CPAP for obstructive sleep apnea. Chest 1993; 103: $1675-1680$

4 Reeves-Hoché MK, Meck R, Zwillich CW: Nasal CPAP. An objective evaluation of patient compliance. Am J Respir Crit Care Med 1994; 149: $149-154$

5 Krieger J, Kurtz D, Petiau Cet al: Long-term compliance with CPAP therapy in obstructive sleep apnea patients and in snorers. Sleep 1996; 19: $136-143$

6 McArdle N, Devereux G, Heidarnejad Het al: Long-term use of CPAP therapy for sleep apnea/hypopnea syndrome. Am J Respir Crit Care Med 1999; 159: 1108 - 1114

7 Krieger J, Kurtz D, Petiau Cet al: Long-term compliance with CPAP therapy in obstructive sleep apnea patients and in snorers. Sleep 1996 19: $136-143$

8 Sin DD, Mayers I, Man GCWet al: Long-term compliance rates to continuous positive airways pressure in obstructive sleep apnea. Chest 2002; $121: 430$ - 435

9 Drake CL, Day R, Hudgel Det al: Sleep during titration predicts continuous positive airway pressure compliance. Sleep 2003; 26: 308 - 311

10 Krieger J, Sforza E, Petiau Cet al: Simplified diagnostic procedure for obstructive sleep apnoea syndrome: lower subsequent compliance with CPAP. Eur Respir J 1998; 12: 776 - 779

11 Means MK, Edinger JD, Husain A: CPAP compliance in sleep apnea patients with and without laboratory titration. Sleep and Breathing 2004; 8: 7-14

12 Ball EM, Banks MB: Determinants of compliance with nasal continuous positive airway pressure treatment applied in a community setting. Sleep Medicine 2001; 2: 195-205

13 Popescu G, Latham M, Allgar Vet al: Continuous positive airway pressure for sleep apnoea/hypopnea syndrome: usefulness of a 2 week trial to identify factors associated with long term use. Thorax 2001; 56: $727-733$ 
14 Pelletier-Fleury N, Rakotonanahary D, Fleury B: The age and other factors in the evaluation of compliance with nasal continuous positive airway pressure for obstructive sleep apnea syndrome. A Cox's proportional hazard analysis. Sleep Med 2001; 2: 225 - 232

15 Lewis KE, Seale L, Bartle IEet al: Early predictors of CPAP use for the treatment of obstructive sleep apnea. Sleep 2004; 27: 134-138

16 Chasens ER, Pack AI, Maislin Get al: Claustrophobia and adherence to CPAP-treatment. West J Nurs Res 2005; 27 (3): 307 - 321

17 Haniffa Met al: The Cochrane Library. Issue 3, http://www.thecochranelibrary.com 2005

18 Fletcher EC, Luckett RA: The effect of positive reinforcement on hourly compliance in nasal continuous positive airway pressure users with obstructive sleep apnea. Am Rev Respir Dis 1991; 143: 936 - 941

19 Wiese HJ, Boethel C, Phillips Bet al: CPAP compliance: video education may help! Sleep Medicine 2005; 6: 171 - 174

20 Aloia MS, Stanchina M, Arnedt JTet al: Treatment adherence and outcomes in flexible vs standard continuous positive airway pressure therapy. Chest 2005; 127: 2085-2093

21 Hoy CJ, Vennelle M, Kingshott RNet al: Can intensive support improve continuous positive airway pressure use in patients with the sleep apnea/hypopnea syndrome? Am J Respir Crit Care Med 1999; 159: $1096-1100$

22 Chervin RD, Theut S, Bassetti Cet al: Compliance with nasal CPAP can be improved by simple interventions. Sleep 1997; 20: 284-289

23 Khanna R, Kline LR: A prospective 8 week trial of nasal interfaces vs. a novel oral interface (Oracle) for treatment of obstructive sleep apnea hypopnea syndrome. Sleep Med 2003; 4: 333-338

24 Massie CA, Hart RW: Clinical outcomes related to interface type in patients with obstructive sleep apnea/hypopnea syndrome who are using continuous positive airway pressure. Chest 2003; 123: 1112 1118

25 Massie CA, Hart RW, Peralez Ket al: Effects of humidification on nasal symptoms and compliance in sleep apnea patients using continuous positive airway pressure. Chest 1999; 116: $403-408$

26 Mador MJ, Krauza M, Pervez Aet al: Effect of heated humidification on compliance and quality of life in patients with sleep apnea using nasal continuous positive airway pressure. Chest 2005; 128: 2151 - 2158

27 Ficker JH, Clarenbach CF, Neukirchner Cet al: Auto CPAP therapy based on the forced oscillation technique. Biomed Technik 2003; 48: 68-72

28 Meurice JC, Marc I, Sériès F: Efficacy of auto-CPAP in the treatment of obstructive sleep apnea/hypopnea syndrome. Am J Respir Crit Care Med 1996; 153: 794-798

29 Konermann M, Sanner B, Vyleta Met al: Use of conventional and selfadjusting nasal continuous positive airway pressure for treatment of severe obstructive sleep apnea syndrome. Chest 1998; 113: 714 - 718

30 Hukins C: Comparative study of autotitrating and fixed-pressure CPAP in the home: a randomized, single-blind crossover trial. Sleep 2004 Dec $15 ; 27(8): 1512-1517$

31 Randerath WJ, Schraeder O, Galetke Wet al: Autoadjusting CPAP therapy based on impedance. Efficacy, compliance and acceptance. Am J Respir Crit Care Med 2001; 163: 652 - 657

32 d'Órtho MP, Grillier-Lanoir V, Lévy Pet al: Constant vs automatic continuous positive pressure therapy. Home evaluation. Chest 2000; 118: $1010-1017$
33 Hudgel DW, Fung C: A long-term randomized, cross-over comparison of auto titrating and standard nasal continuous airway pressure. Sleep 2000; $23: 1-4$

34 Massie CA, McArdle N, Hart RWet al: Comparison between automatic and fixed positive airway pressure therapy in the home. Am J Respir Crit Care Med 2003; 167: 20-23

35 Gay PC, Herold DL: A randomized, double-blind clinical trial comparing continuous positive airway pressure with a novel bilevel pressure system for treatment of obstructive sleep apnea syndrome. Sleep 2003; 26: $864-869$

36 Reeves-Hoché MK, Hudgel DW, Meck Ret al: Continuous versus bilevel positive airway pressure for obstructive sleep apnea. Am J Respir Crit Care Med 1995; 151: 443 - 449

37 Doherty LS, Kiely JL, Swan Vet al: Long-term effects of nasal continuous positive airway pressure on cardiovascular outcomes in sleep apnea syndrome. Chest 2005; 127: 2076-2084

38 Marin JM, Carrizo SJ, Vicente Eet al: Long-term cardiovascular outcomes in men with obstructive sleep apnea-hypopnoea with or without treatment with continuous positive airway pressure: an observational study. Lancet 2005; 365: 1046 - 1053

39 Orth $M$, Duchna HW, Leidag Met al: Driving simulator performance and neuropsychological testing in OSAS before and under CPAP. Eur Respir J 2005; 26: 898 - 903

40 Rasche K, Orth M, Duchna HWet al: CPAP therapy in obstructive sleep apnea syndrome. Side effects and acceptance in long-term follow-up. Med Klin 1994; 89: 86-88

41 Waldhorn RE, Herrick T, Nguyen MCet al: Long-term compliance with nasal continuous positive airway pressure therapy of obstructive sleep apnea. Chest 1990; 97: $33-38$

42 Akashiba T, Kurashina K, Sasaki Iet al: Long-term effects and complications of home nasal CPAP therapy for obstructive sleep apnea patients. Nippon Kyobu Shikkan Gakkai Zasshi 1992; 30: 604-608

43 Pépin JL, Leger P, Veale Det al: Side effects of nasal continuous positive airway pressure in sleep apnea syndrome. Study of 193 patients in two french Sleep Centers. Chest 1995; 107: 375 - 381

44 Hoffstein V, Viner S, Mateika Set al: Treatment of obstructive sleep apnea with nasal continuous positive airway pressure. Am Rev Respir Dis 1992; 145: $841-845$

45 Engleman HM, Asgari-Jirhandeh N, McLeod ALet al: Self-reported use of CPAP and benefits of CPAP-therapy. Chest 1996; 109: 1470-1476

46 Barnes $M$, Houston D, Worsnop CJet al: A randomized controlled trial of continuous positive airway pressure in mild obstructive sleep apnea. Am J Respir Crit Care Med 2002; 165: 773 - 780

47 Engleman HM, Martin SE, Douglas NJ: Compliance with CPAP therapy in patients with the sleep apnoea/hypopnea syndrome. Thorax 1994 49: $263-266$

48 Pépin JL, Krieger JL, Rodenstein Det al: Effective compliance during the first 3 months of continuous positive airway pressure. A European prospective study of 121 patients. Am J Respir Crit Care Med 1999; 160: $1124-1129$

49 Hui DS, Choy DK, Li TSet al: Determinants of continuous positive airway pressure compliance in a group of Chinese patients with obstructive sleep apnea. Chest 2001; 120: 170-176

50 Meurice JC, Dore P, Paquereau Jet al: Predictive factors of long-term compliance with nasal continuous positive airway pressure treatment in sleep apnea syndrome. Chest 1994; 105: 429-433 